\title{
Synergistic effects of cisplatin and proteasome inhibitor bortezomib on human bladder cancer cells
}

\author{
ECE KONAC ${ }^{1}$, NURAY VAROL ${ }^{1}$, ILKER KILICCIOGLU $^{1}$ and CENK Y. BILEN ${ }^{2}$ \\ ${ }^{1}$ Department of Medical Biology and Genetics, Faculty of Medicine, Gazi University, Ankara 06500; \\ ${ }^{2}$ Department of Urology, Faculty of Medicine, Hacettepe University, Ankara 06100, Turkey
}

Received November 3, 2014; Accepted May 8, 2015

DOI: $10.3892 / \mathrm{ol} .2015 .3250$

\begin{abstract}
The proteasome inhibitor bortezomib is a promising novel agent in bladder cancer therapy; however, inducible cytoprotective mechanisms may limit its potential efficacy. To date, the cellular and molecular effects of proteasome inhibitors on bladder cancer cells have been poorly characterized. Despite the consistent rate of initial responses, cisplatin treatment typically results in the development of chemoresistance, leading to therapeutic failure. Therefore, the present study aimed to characterize the molecular mechanisms underlying the anti-proliferative effects of cisplatin and bortezomib combination therapy on the human T24 bladder cancer cell line, by analyzing the protein expression levels of apoptotic genes. Cytotoxic effects were measured using a water-soluble tetrazolium salt-1 assay, and the apoptosis-associated molecules were examined using western blot analysis and ELISA. It was observed that combined administration of cisplatin and bortezomib induced upregulation of caspase-3, -8 and -9, B-cell lymphoma-2 (Bcl-2)-like 11 and Bcl-2-interacting killer, but downregulated Bcl-2 and Bcl-extra large protein expression levels in T24 cells in a dose-dependent manner. Furthermore, enhanced protein expression of caspase- 8 and -9 , in line with the significantly increased caspase-3 activation, was detected when the cells were treated with a combination of cisplatin and bortezomib, compared with that of either agent alone. Bortezomib appeared to synergize with cisplatin to promote apoptosis via the extrinsic and intrinsic apoptotic pathways. Taken together, the results of the current study provide the preclinical framework for additional evaluation of the effects of combining bortezomib with other agents to induce apoptosis in bladder cancer cells.
\end{abstract}

Correspondence to: Professor Ece Konac, Department of Medical Biology and Genetics, Faculty of Medicine, Gazi University, Beşevler, Ankara 06500, Turkey

E-mail: ecemercanoglu@yahoo.com

Key words: apoptosis, bladder cancer cells, bortezomib, cisplatin

\section{Introduction}

Systemic chemotherapy is one of the primary modalities used to improve the survival of patients with metastatic urothelial cancer (UC). Cisplatin-based chemotherapy remains the standard treatment strategy for patients with metastatic UC; however, despite the administration of various novel combination regimens, the overall response rate varies between 36-69\%. Another limiting factor associated with the currently available chemotherapeutic regimens is their level of toxicity. As such, the treatment of metastatic UC with cytotoxic chemotherapy has reached a therapeutic plateau, and the identification of novel treatment modalities is urgently required (1).

Cisplatin is an effective antitumor agent owing to its ability to induce intra- and inter-strand DNA cross-links (2). Cisplatin-based combination chemotherapy is currently the primary treatment strategy for patients with advanced bladder cancer, however, its clinical use as an anti-cancer agent is predominantly limited by its association with a high incidence of chemoresistance $(3,4)$. One of the known mechanisms underlying chemoresistance is alteration of the apoptotic signaling pathways and a resultant decreased apoptotic response (5). For example, B-cell lymphoma-2 (Bcl-2) is a significant anti-apoptotic protein, responsible for regulation of the mitochondrial apoptotic signaling pathway. Overexpression of Bcl-2 is known to block apoptosis by preventing translocation of Bcl-2-associated X protein to the mitochondrial membrane and reducing programmed cell death (3). Bcl-2 is frequently upregulated in multiple types of cancer, including bladder cancer (6). Furthermore, upregulated $\mathrm{Bcl}-2$ protein appears to be crucial in the development of cisplatin resistance $(3,7)$. To overcome this resistance, improvements in systemic combined chemotherapeutic regimes and the development of novel treatment regimens are essential. For example, combining cisplatin with other agents is known to enhance its efficiency. These combinations may result in decreased levels of anti-apoptotic protein expression and upregulation of pro-apoptotic protein expression by shifting the balance between cell death and survival (7). Various studies have demonstrated that proteasome inhibition is the key regulator of intracellular protein degradation $(8,9)$. This inhibition promotes the degradation of anti-apoptotic proteins while preventing the degradation of pro-apoptotic proteins, resulting in increased accumulation of pro-apoptotic proteins within the cells, and subsequent cell 
growth inhibition and programmed cell death in numerous malignant cell types $(8,9)$.

Bortezomib is a potent, selective and reversible inhibitor of the $26 \mathrm{~S}$ proteasome, comprised of a complex multi-subunit protease that controls the degradation of short-lived regulatory proteins involved in various cellular processes, including apoptosis $(9,10)$. Previous studies have identified that the bortezomib-induced apoptotic mechanism is the key regulator in the balance of pro- and anti-apoptotic Bcl-2 family proteins $(9,11)$. Bortezomib treatment upregulates the expression of pro-apoptotic proteins, including Bcl-2-like 11 (Bim) and Bcl-2-interacting killer (Bik), and downregulates the expression of anti-apoptotic Bcl-2 family proteins, for example Bcl-2 (11).

The present study examined the anti-proliferative effects of cisplatin and bortezomib, applied alone or in combination, on the human T24 urinary bladder carcinoma cell line. Following treatment with cisplatin and bortezomib alone or in combination, the activation of caspase-3, -8 and -9 , and the expression levels of anti-apoptotic [Bcl-2 and Bcl-extra large (Bcl-xL)] and pro-apoptotic (Bim and Bik) proteins were investigated. Furthermore, the current study aimed to establish whether the synergistic effects of combined cisplatin and bortezomib treatment may offer a potential therapeutic approach to overcome cisplatin resistance.

\section{Materials and methods}

Cell lines and chemicals. The human T24 urinary bladder carcinoma cell line was obtained from the American Type Culture Collection (Manassas, VA, USA). The T24 cells were cultured in McCoy's 5A medium containing L-glutamine, $10 \%$ fetal bovine serum, $100 \mathrm{U} / \mathrm{ml}$ penicillin and $100 \mathrm{mg} / \mathrm{ml}$ streptomycin (Thermo Fisher Scientific, Inc., Waltham, MA, USA), and incubated in a humidified atmosphere of $5 \%$ $\mathrm{CO}_{2}$ at a temperature of $37^{\circ} \mathrm{C}$. Cisplatin was obtained from Sigma-Aldrich (St. Louis, MO, USA) and bortezomib was obtained from BioVision, Inc. (Milpitas, CA, USA)

Cell viability assay. The antitumor effects of single and combined cisplatin and bortezomib treatment on the viability of T24 cells were determined by performing cell proliferation water-soluble tetrazolium salt-1 (WST-1) assays (Roche Diagnostics GmbH, Mannheim, Germany). Half maximal inhibitory concentration values for cisplatin and bortezomib were determined by treating cells with cisplatin $(0,0.5,2.5$, 5, 10 and $20 \mu \mathrm{M})$ and/or bortezomib $(0,1,5,7.5,10,25,50$, 100,200 and $300 \mathrm{nM}$ ). The control cells were treated only with cell culture medium. Briefly, in each well of a 96-well plate, $5 \times 10^{3}$ cells were seeded in $200 \mu \mathrm{l}$ medium and treated with cisplatin, bortezomib or a combination of the two agents for $24 \mathrm{~h}$. WST-1 solution $(10 \mu \mathrm{l})$ was added to each well and absorbance was measured after $3 \mathrm{~h}$ at a wavelength of $450 \mathrm{~nm}$ using an ELISA reader (Spectramax ${ }^{\circledR} \mathrm{M} 3$; Molecular Devices LLC, Sunnyvale, CA, USA) following the incubation period.

Active caspase-3 level. Caspase-3 protein activity was measured using a luminescence assay, according to the manufacturer's instructions [PathScan ${ }^{\circledR}$ Cleaved Caspase-3 (Asp175) Sandwich ELISA kit; Cell Signaling Technology, Inc. (Danvers,
MA, USA)]. Considering that the caspase family of proteases have key effector roles in apoptosis in mammalian cells, the aim was to detect the pro-apoptotic effects of treatment with cisplatin and bortezomib alone or in combination for $24 \mathrm{~h}$. Briefly, addition of the reagent to the wells induced cell lysis, followed by caspase cleavage of the substrate and generation of a luminescent signal by luciferase that was proportional to the level of caspase activity. Caspase- 3 activity was quantified by reading the absorbance at a wavelength of $450 \mathrm{~nm}$ using a microplate ELISA reader.

Protein extraction and western blot analysis. Western blot analysis of Bcl-2, Bcl-xL, Bim, Bik, caspase- 8 and caspase-9 was performed as previously described (12). Briefly, cells were lysed in lysis buffer (Cell Signaling Technology, Inc.) containing $1 \mathrm{mM}$ phenylmethanesulfonylfluoride (Sigma-Aldrich) prior to treatment with the specified concentrations of cisplatin and/or bortezomib for $24 \mathrm{~h}$. Equal quantities of protein were loaded and separated by $12 \%$ SDS-PAGE then transferred to a polyvinylidene difluoride membrane (Thermo Fisher Scientific, Inc.). Following blocking with $5 \% \mathrm{w} / \mathrm{v}$ non-fat milk or $5 \% \mathrm{w} / \mathrm{v}$ bovine serum albumin in Tris-buffered saline with $0.1 \%$ Tween 20 (TBST-T), the membrane was incubated overnight at $4^{\circ} \mathrm{C}$ with rabbit anti-human $\mathrm{Bcl}-2$ (catalog no. PA5-27094), Bcl-xL (catalog no. PA5-17805), Bim (catalog no. PA5-11385), Bik (catalog no. PA5-20249), caspase-8 (catalog no. PA5-20118) and caspase-9 (catalog no. PA5-19904) (Thermo Fisher Scientific, Inc.) polyclonal antibodies, as well as rabbit anti-human $\beta$-actin monoclonal antibody (catalog no. 4970; Cell Signaling Technology, Inc.) as the loading control. All primary antibodies were diluted 1:1,000. This process was followed by incubation with goat anti-rabbit horseradish peroxidase (HRP)-conjugated secondary antibody (catalog no. 31210; dilution, 1:5,000; Thermo Fisher Scientific, Inc.) for $2 \mathrm{~h}$ at room temperature. Proteins were visualized using a Kodak Gel Logic 2200 imaging system (Kodak, Rochester, NY, USA) with Luminata ${ }^{\mathrm{TM}}$ Crescendo Western HRP substrate (EMD Millipore, Billerica, MA, USA).

Statistical analysis. Each data point was measured in three independent experiments. Cell viability was analyzed by performing one-way analysis of variance and multiple comparison analyses were performed using SPSS software (version 15.0; SPSS, Inc., Chicago, IL, USA). $\mathrm{P}<0.01$ was considered to indicate a statistically significant difference and the results are expressed as the mean \pm standard deviation.

\section{Results}

Combined treatment with cisplatin and bortezomib enhances inhibition of T24 cell proliferation. Optimal doses of cisplatin alone, bortezomib alone, and cisplatin and bortezomib combined were determined by performing a WST-1 assay. To identify the effects of exposure to cisplatin and bortezomib alone and in combination, T24 cells were treated with various concentrations of cisplatin $(0-20 \mu \mathrm{M})$ and bortezomib (0-300 $\mathrm{nM})$ for $24 \mathrm{~h}$. The most effective and least toxic doses of cisplatin (Fig. 1A) and bortezomib (Fig. 1B) alone were determined to be $10 \mu \mathrm{M}$ and $10 \mathrm{nM}$, respectively. Furthermore, the most effective doses of cisplatin and bortezomib during their 

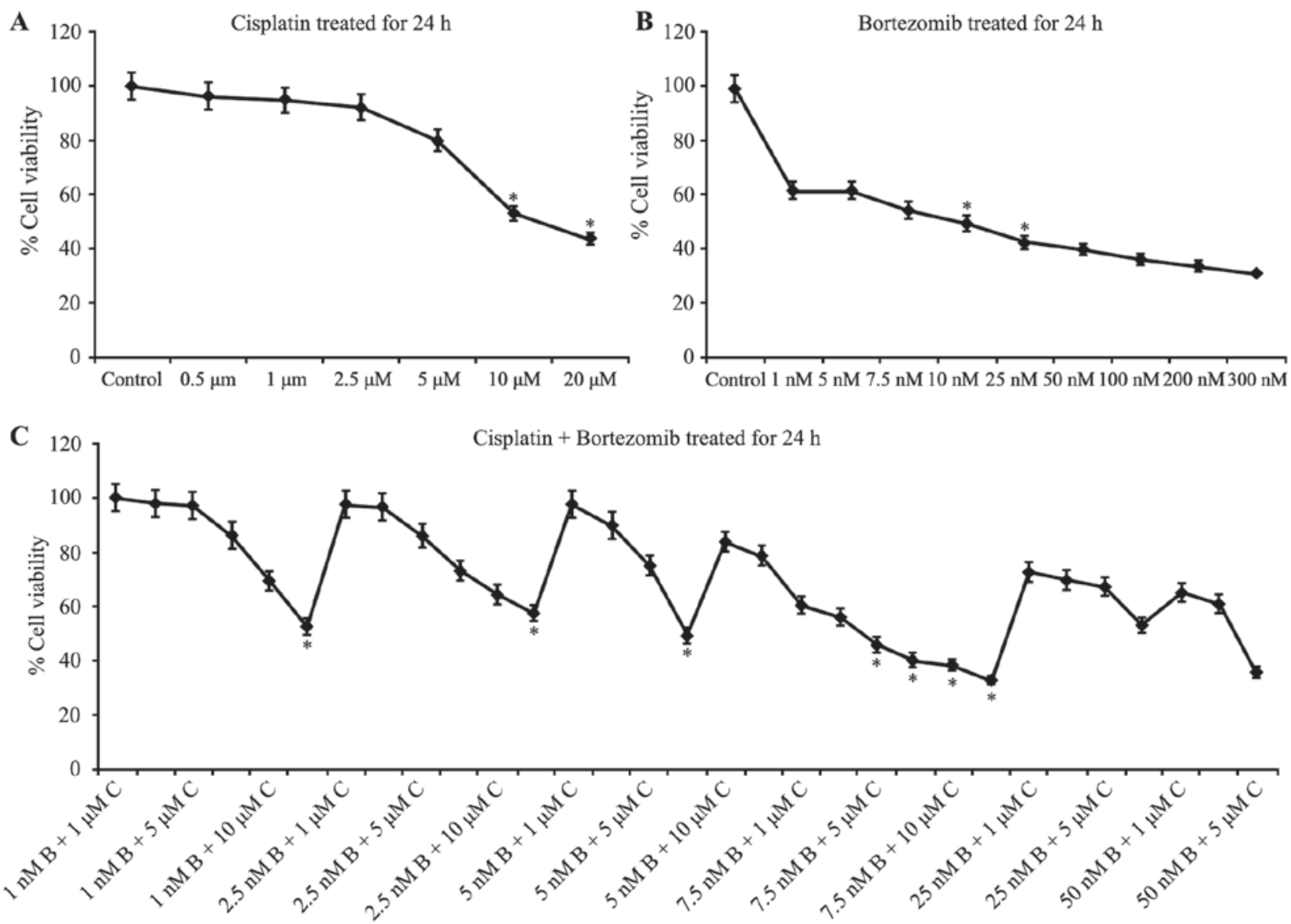

Figure 1. Combined treatment with cisplatin and bortezomib inhibits cell proliferation of T24 cells. Graphical representation of water soluble tetrazolium salt assay, indicating percentage change in cell viability in T24 cells treated with (A) cisplatin $(0,0.5,1,2.5,5,10$ and $20 \mathrm{mM}),(\mathrm{B})$ bortezomib $(0,1,5,7.5,10$ 25, 50, 100, 200 and $300 \mathrm{nM}$ ) and (C) cisplatin plus bortezomib for $24 \mathrm{~h}$. Data points represent the mean \pm standard deviation of triplicate experiments. "IC $\mathrm{C}_{50}$ value. C, cisplatin; B, bortezomib.

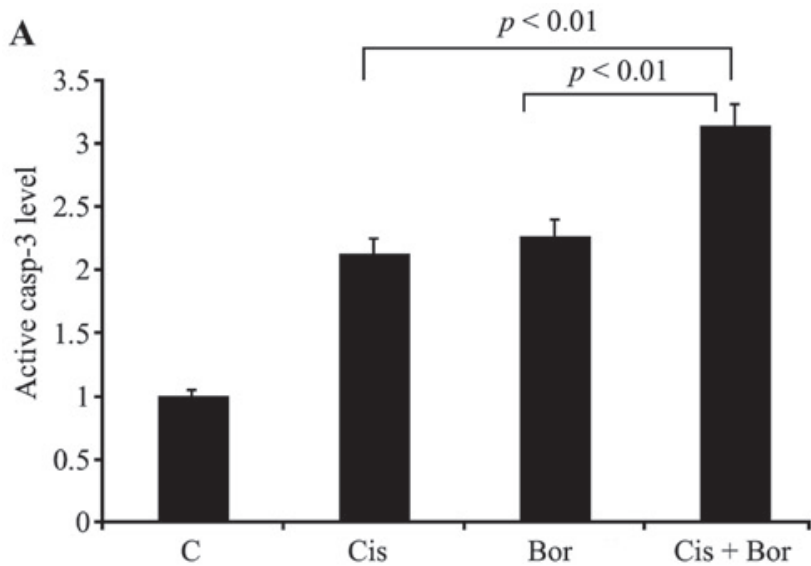

B

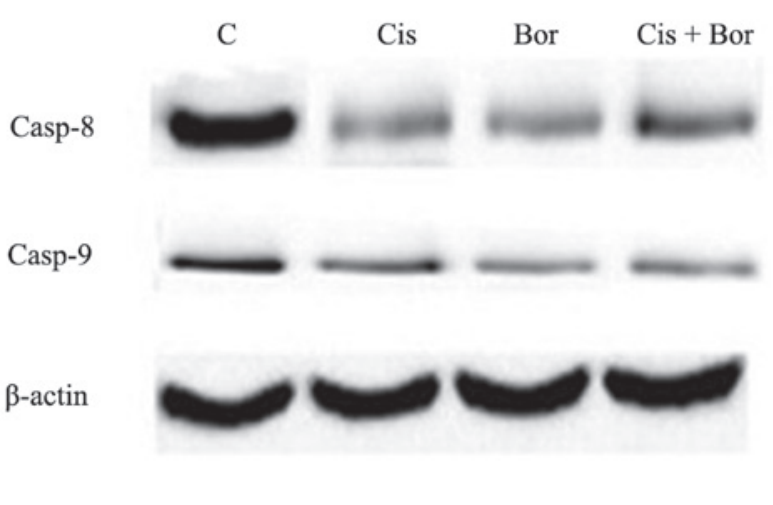

Figure 2. Combined cisplatin and bortezomib treatment enhances casp-3, -8 and -9 activity. (A) Expression levels of effector casp-3 protein, determined by ELISA. Values are expressed as the mean \pm standard deviation of triplicate experiments. (B) Expression levels of initiator apoptotic caspases, detected by western blotting. Casp, caspase; C, control; cis, cisplatin; Bor, bortezomib.

combined use were $7.5 \mu \mathrm{M}$ and $5 \mathrm{nM}$, respectively (Fig. 1C). These values indicated that the two agents synergistically enhance cell proliferation inhibition.

Combined treatment with cisplatin and bortezomib enhances caspase- $-3,-8$ and -9 activity. Treatment of T24 cells with $10 \mu \mathrm{M}$ cisplatin, $10 \mathrm{nM}$ bortezomib and cisplatin plus bortezomib (7.5 $\mu \mathrm{M}$ and $5 \mathrm{nM})$ resulted in 2.1-, 2.3- and 3.2 -fold increases in caspase- 3 activation, respectively. The cause of these significant increases was suggested to be the triggering of cell apoptosis $(\mathrm{P}<0.01$; Fig. $2 \mathrm{~A})$. This apoptosis was associated with more effective induction caspase-3 activation by cisplatin and bortezomib combination treatment of T24 cells, compared with that of the administration of 
A

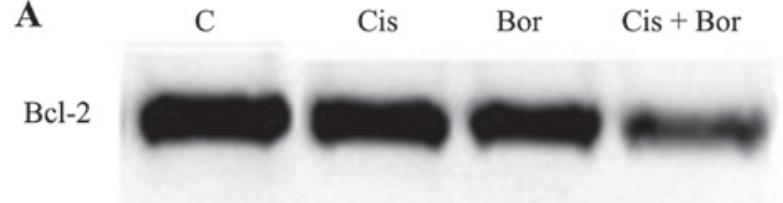

Bcl- $\mathrm{X}_{\mathrm{L}}$

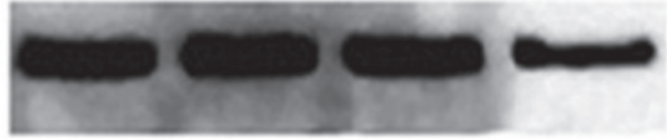

$\beta$-actin

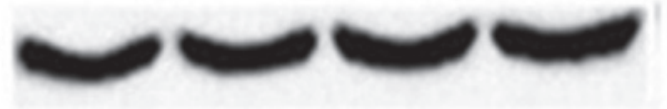

B

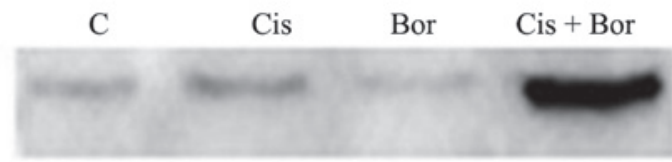

Bik

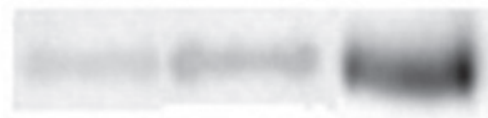

$\beta$-actin

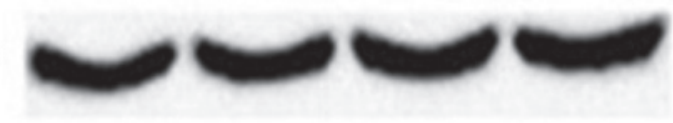

Figure 3. Combined treatment downregulates the expression of anti-apoptotic proteins and enhances the expression of pro-apoptotic proteins. Western blot analysis results of (A) anti-apoptotic and (B) pro-apoptotic protein expression $24 \mathrm{~h}$ following cis, bor and cis plus bortezomib combination treatment. C, control; cis, cisplatin; bor, bortezomib; Bcl-2, B-cell lymphoma-2; Bcl-xL, B-cell lyphoma-extra large; Bim, B-cell lyphoma-2-like 11; Bik, Bcl-2-interacting killer.

the two agents alone. Furthermore, enhanced activation of caspase- 8 and -9 were detected when the cells were treated with cisplatin and bortezomib in combination, in agreement with the effects on caspase-3 activation (Fig. 2B).

Combined treatment with cisplatin and bortezomib alters the balance of Bcl-2 family protein expression levels. The present study also investigated the effect of cisplatin and bortezomib treatment on the expression levels of the pro-apoptotic and anti-apoptotic proteins of the Bcl-2 family. Overexpression of $\mathrm{Bcl}-2$ protein is associated with the development of cisplatin resistance, with $\mathrm{Bcl}-2$ and $\mathrm{Bcl}-\mathrm{xL}$ protein expression levels known to be high in cisplatin-resistant T24 cells. Western blot analysis revealed that the expression levels of Bcl-2 and Bcl-xL were not markedly altered following treatment of T24 cells with cisplatin or bortezomib alone, compared with those of the control. However, combined administration of these agents resulted in markedly decreased expression of Bcl-2 and Bcl-xL at the protein level (Fig. 3A). Furthermore, it was demonstrated that, following treatment with bortezomib alone, only the expression of pro-apoptotic protein Bik increased. However, combined administration of cisplatin and bortezomib led to an increase in the expression of pro-apoptotic proteins Bik and Bim (Fig. 3B). These results indicated that combined administration of cisplatin and bortezomib may synergistically induce apoptosis. In addition, exposure to a lower-dose drug combination resulted in an anti-proliferative effect.

\section{Discussion}

Preclinical studies employing bladder cancer cells have demonstrated that combined therapy with conventional agents may result in greater tumor growth inhibition than that observed following therapy with either agent alone, without inducing significant increases in toxicity (13-15). The proteasome inhibitor bortezomib is a promising novel agent in the treatment of bladder cancer; however, inducible cytoprotective mechanisms may limit its potential efficacy (15). In previous studies, bortezomib has been observed to enhance the activity of cisplatin, particularly in cisplatin-resistant cells $(6,8,16-18)$. The present study demonstrated for the first time that cisplatin and bortezomib combined treatment induced inhibition of cell proliferation by intrinsic and extrinsic apoptotic signaling pathways in the T24 human bladder cancer cell line. Exposure to a lower-dose drug combination resulted in a significant anti-proliferative effect. The results revealed that cisplatin plus bortezomib combination treatment was more potent than the administration of either agent alone.

The development of resistance to treatment is one of the major limitations to successful cisplatin-based chemotherapy regimes, frequently resulting in poor clinical prognoses. Chemoresistance has previously been associated with the failure of cisplatin to induce apoptosis $(3,19)$, the most common response of cells to chemotherapeutic agents. To date, two major apoptotic pathways have been identified in mammalian cells. The first involves caspase-8, which is activated by membrane death receptor-mediated extrinsic signaling pathways, while the second involves mitochondria-dependent intrinsic signaling pathways, characterized by the activation of caspase- 9 by cytochrome $c$ release into the cytosol and subsequent apoptosome formation. These extrinsic and intrinsic apoptotic signaling pathways converge at the level of caspase-3 activation (20). The current study demonstrated that the extrinsic death receptor- and intrinsic mitochondria-dependent signaling pathways were activated following combinatorial treatment of T24 cells with cisplatin and bortezomib. Furthermore, caspases-3, -8 and -9 only exhibited marked activation following combined treatment. Thus, increased caspase activation indicates that combined treatment with these agents induces cell apoptosis. Bortezomib appeared to enhance apoptosis and inhibit proliferation in T24 cells, as well as enhance the growth-inhibitory effects of cisplatin. The current results corroborate those of previous studies performed in various other cell types evaluating the effect of bortezomib treatment on caspase activation and apoptosis $(21,22)$.

Although caspase activation has a key role in the mechanism of apoptosis, the primary controllers underlying caspase activation are the Bcl-2 family proteins (23). $\mathrm{Bcl}-2$ protein family members function as key regulators of cellular apoptosis and are important determinants of cellular sensitivity or resistance to chemotherapeutic agents (7), with overexpression of $\mathrm{Bcl}-2$ known to block apoptosis. An association between Bcl-2 upregulation and cisplatin resistance was previously reported in a cisplatin-resistant subclone of the human T24 bladder cancer cell line $(3,6)$. Therefore, Bcl-2 may be a significant target for the prevention of resistance to cisplatin treatment. It is known that 
the expression levels of pro-apoptotic and anti-apoptotic Bcl-2 family proteins are directly regulated by proteasome inhibition. Therefore, the expression levels of Bcl-2 family proteins may change in response to inhibitors of the ubiquitin proteasome system $(24,25)$. Western blot analyses performed in the present study identified no noticeable alterations in the expression levels of $\mathrm{Bcl}-2$ and $\mathrm{Bcl}-\mathrm{xL}$ following treatment with cisplatin and bortezomib alone, when compared with those of the control. However, the expression levels of Bcl-2 and Bcl-xL were markedly decreased following combination treatment. Furthermore, it was demonstrated that only the expression of pro-apoptotic protein Bik was increased following treatment with bortezomib alone. By contrast, combination treatment resulted in increased expression levels of the two pro-apoptotic proteins Bik and Bim. This accumulation of Bik and Bim indicated the synergistic action of cisplatin and bortezomib on the induction of apoptosis.

In conclusion, the results of the current study demonstrated the potential of bortezomib and cisplatin combination therapy in the treatment of bladder cancer. This effect occurred via the efficient activation of intrinsic and extrinsic apoptotic signaling pathways in $\mathrm{T} 24$ cells, by modulation of the balance among Bcl-2 family proteins towards apoptosis. This synergistic effect of combined agents may offer a novel approach to overcome cisplatin resistance, however, additional research using in vivo models is required.

\section{References}

1. Latini DM, Lerner SP, Wade SW, Lee DW and Quale DZ: Bladder cancer detection, treatment and outcomes: Opportunities and challenges. Urology 75: 334-339, 2010.

2. da Silva GN, de Castro Marcondes JP, de Camargo EA, da Silva Passos Júnior GA, Sakamoto-Hojo ET and Salvadori DM: Cell cycle arrest and apoptosis in TP53 subtypes of bladder carcinoma cell lines treated with cisplatin and gemcitabine. Exp Biol Med 235: 814-824, 2010.

3. Yu HM and Wang TC: Mechanism of cisplatin resistance in human urothelial carcinoma cells. Food Chem Toxicol 50: 1226-1237, 2012.

4. Galluzzi L, Senovilla L, Vitale I, Michels J, Martins I, Kepp O, Castedo M and Kroemer G: Molecular mechanisms of cisplatin resistance. Oncogene 31: 1869-1883, 2012.

5. Köberle B, Tomicic MT, Usanova S and Kaina B: Cisplatin resistance: Preclinical findings and clinical implications. Biochim Biophys Acta 1806: 172-182, 2010.

6. Cho HJ, Kim JK, Kim KD, et al: Upregulation of Bcl-2 is associated with cisplatin-resistance via inhibition of Bax translocation in human bladder cancer cells. Cancer Lett 237: 56-66, 2006.

7. Li C, Li R, Grandis JR and Johnson DE: Bortezomib induces apoptosis via Bim and Bik up-regulation and synergizes with cisplatin in the killing of head and neck squamous cell carcinoma cells. Mol Cancer Ther 7: 1647-1655, 2008.

8. Al-Eisawi Z, Beale P, Chan C, Yu JQ and Huq F: Carboplatin and oxaliplatin in sequenced combination with bortezomib in ovarian tumour models. J Ovarian Res 6: 78, 2013.
9. Chen D, Frezza M, Schmitt S, Kanwar J and Dou QP: Bortezomib as the first proteasome inhibitor anticancer drug: Current status and future perspectives. Curr Cancer Drug Targets 11: 239-253, 2011.

10. Hutter G, Rieken M, Pastore A, Weigert O, Zimmermann Y, Weinkauf M, Hiddemann W and Dreyling M: The proteasome inhibitor bortezomib targets cell cycle and apoptosis and acts synergistically in a sequence dependent way with chemotherapeutic agents in mantle cell lymphoma. Ann Hematol 91: 847-856, 2012.

11. Yang TM, Barbone D, Fennell DA and Broaddus VC: Bcl-2 family proteins contribute to apoptotic resistance in lung cancer multicellular spheroids. Am J Respir Cell Mol Biol 41: 14-23, 2009.

12. Konac E, Varol N, Yilmaz A, Menevse S and Sozen S: DNA methyltransferase inhibitor-mediated apoptosis in the Wnt/ $\beta$-catenin signal pathway in a renal cell carcinoma cell line. Exp Biol Med (Maywood) 238: 1009-1016, 2013.

13. Kamat AM, Karashima T, Davis DW, Lashinger L, Bar-Eli M, Millikan R, Shen Y, Dinney CP and McConkey DJ: The proteasome inhibitor bortezomib synergizes with gemcitabine to block the growth of human 253JB-V bladder tumors in vivo. Mol Cancer Ther 3: 279-290, 2004.

14. Papageorgiou A, Kamat A, Benedict WF, Dinney C and McConkey DJ: Combination therapy with IFN-alpha plus bortezomib induces apoptosis and inhibits angiogenesis in human bladder cancer cells. Mol Cancer Ther 5: 3032-3041, 2006.

15. Qi W, White MC, Choi W, Guo C, Dinney C, McConkey DJ and Siefker-Radtke A: Inhibition of inducible heat shock protein-70 (hsp72) enhances bortezomib-induced cell death in human bladder cancer cells. PLoS One 8: e69509, 2013.

16. Yerlikaya A, Altıkat S, Irmak R, Cavga FZ, Kocacan SA and Boyaci I: Effect of bortezomib in combination with cisplatin and 5 fluorouracil on 4T1 breast cancer cells. Mol Med Rep 8: 277-281, 2013.

17. Fribley AM, Evenchik B, Zeng Q, Park BK, Guan JY, Zhang H, Hale TJ, Soengas MS, Kaufman RJ and Wang CY: Proteasome inhibitor PS-341 induces apoptosis in cisplatin-resistant squamous cell carcinoma cells by induction of Noxa. J Biol Chem 281: 31440-31447, 2006.

18. Brozovic A, Ambriović-Ristov A and Osmak M: The relationship between cisplatin-induced reactive oxygen species, glutathione, and BCL-2 and resistance to cisplatin. Crit Rev Toxicol 40: 347-359, 2010.

19. Rabik CA and Dolan ME: Molecular mechanisms of resistance and toxicity associated with platinating agents. Cancer Treat Rev 33: 9-23, 2007.

20. Hyman BT and Yuan J: Apoptotic and non-apoptotic roles of caspases in neuronal physiology and pathophysiology. Nat Rev Neurosci 13: 395-406, 2012.

21. Kim SY, Song X, Zhang L, Bartlett DL and Lee YJ: Role of Bcl-xL/Beclin-1 in interplay between apoptosis and autophagy in oxaliplatin and bortezomib-induced cell death. Biochem Pharmacol 88: 178-188, 2014.

22. Krętowski R, Borzym-Kluczyk M and Cechowska-Pasko M: Efficient induction of apoptosis by proteasome inhibitor: Bortezomib in the human breast cancer cell line MDA-MB-231. Mol Cell Biochem 389: 177-185, 2014.

23. Elkholi R, Floros KV and Chipuk JE: The role of BH3-only proteins in tumor cell development, signaling, and treatment. Genes Cancer 2: 523-537, 2011.

24. Fennell DA, Chacko A and Mutti L: BCL-2 family regulation by the $20 \mathrm{~S}$ proteasome inhibitor bortezomib. Oncogene 27: 1189-1197, 2008

25. Neutzner A,LiS, Xu S and Karbowski M: The ubiquitin/proteasome system-dependent control of mitochondrial steps in apoptosis. Semin Cell Dev Biol 23: 499-508, 2012. 